\title{
TITLE:
}

\section{Linear stability of steady zonal jet flows induced by a small-scale forcing on a plane}

\author{
AUTHOR(S):
}

Obuse, Kiori; Takehiro, Shin-ichi; Yamada, Michio

\section{CITATION:}

Obuse, Kiori ... [et al]. Linear stability of steady zonal jet flows induced by a small-scale forcing on a plane. Physica D: Nonlinear Phenomena 2011, 240(22): 1825-1834

\section{ISSUE DATE:}

2011-11

URL:

http://hdl.handle.net/2433/151132

\section{RIGHT:}

(C) 2011 Elsevier B.V.; This is not the published version. Please cite only the published version.; この論文は出版社版でありません。引用の際に は出版社版をご確認ご利用ください。 


\title{
Linear stability of steady zonal jet flows induced by a small-scale forcing on a $\beta$ plane
}

\author{
Kiori Obuse, Shin-ichi Takehiro ${ }^{\dagger}$ and Michio Yamada ${ }^{\ddagger}$ \\ Research Institute for Mathematical Sciences, Kyoto University, \\ Kyoto, 606-8502, Japan
}

November 27, 2011

\begin{abstract}
We analytically obtain steady isolated zonal jet solutions of the evolution equation of zonal flows on a $\beta$ plane with a homogeneous zonal flow and a small-scale sinusoidal transversal flow in the background, derived by Manfroi and Young (1999). It is shown that these steady zonal jet solutions are all linearly unstable. Numerical time integrations of the evolution equation also confirm that the perturbed unstable steady solution becomes a uniform flow in the long run. These results suggest that mergers/disappearances of zonal jets superposed upon background forced two-dimensional turbulence on a $\beta$ plane or a rotating sphere might be due to the intrinsic instability of the zonal jets.
\end{abstract}

Keywords: rotating fluid, barotropic flow, zonal jet, turbulence, beta effect, Cahn-Hilliard equation

*Corresponding author. Postal address: Research Institute for Mathematical Sciences, Kyoto University, Kyoto, 606-8502, Japan; Tel.: +81-75-753-7221; Fax: +81-75-753-7272; E-mail address: obuse@kurims.kyoto-u.ac.jp

${ }^{\dagger}$ E-mail address: takepiro@gfd-dennou.org

${ }_{\ddagger}^{\ddagger}$ E-mail address: yamada@kurims.kyoto-u.ac.jp 


\section{Introduction}

In contrast to three-dimensional turbulence, it is well known that two-dimensional turbulence in an irrotational system is characterized by the inverse energy cascade (Kraichinan [7]). The kinetic energy injected at a small scale is transferred to larger scales, which brings about an isotropic larger-scale structure as time progresses. The effect of rotation, which is so-called the $\beta$ effect, retards this inverse energy cascade and causes anisotropic features. A pioneering numerical study of two-dimensional turbulence on a $\beta$ plane by Rhines [15] shows that a multiple zonal band structure emerges as the turbulent motion evolves. It is explained that kinetic energy transferred from a small scale is arrested around the Rhines scale, where the $\beta$ effect and the advective effect in the governing equation become comparable, and then the fluid motion is governed by the zonal flows whose width is roughly the same as the Rhines scale. Many succeeding studies have confirmed the emergence of the multiple zonal-band structure on both a $\beta$ plane and a rotating two-dimensional sphere when turbulent motion is driven by a small-scale stochastic forcing (e.g. Williams [18], Vallis and Maltrud [17], Nozawa and Yoden [13], Huang and Robinson [5]).

A recent numerical experiment of forced two-dimensional turbulence on a rotating sphere shows, however, that when the time integration is carried much further than the previous studies, multiple zonal flows merge passing over the Rhines scale and large scale two or three alternating zonal jets remain at the final stage (Obuse et al. [14]). Mergers of zonal jets and emergence of a zonal structure larger than the Rhines scale are also observed when the small-scale forcing is a white noise type instead of the Markovian type explained in Nozawa and Yoden [13] and Obuse et al. [14] (Huang et al. [6]).

One of the possible interpretations of such a merger of zonal jets is that the state with multiple zonal jets may be dynamically unstable and the transition to a stable state with wider and fewer zonal jets may occur. It is accordingly tempted to examine the stability of zonal jets driven and maintained by a small-scale forcing and background small-scale turbulent motions.

However, it is difficult to investigate the stability of zonal flows induced by a small-scale stochastic forcing, because it is hard to construct a tractable and reasonable physical configuration. We therefore investigate the stability of zonal flows induced by a small-scale deterministic forcing instead of a stochastic forcing as a first step. Manfroi and Young [10] investigated the evo- 
lution of zonal flows on a $\beta$ plane when there is a homogeneous zonal flow and a small-scale sinusoidal transversal flow as a background base flow. They derived a time evolution equation of zonal flows with a small-scale background flow by using a multiple-scale expansion technique. This equation is a special case of Cahn-Hilliard equation [3], and we call it Manfroi-Young equation for clarity. From numerical experiments of the Manfroi-Young equation, when the bottom drag is absent, a multiple zonal band structure, i.e. the structure with multiple zonal jets emerges, and the gradual disappearances of the zonal jet occur one by one, forming a thin prograde jet and a broad retrograde jet in the considered periodic domain. They also pointed out that the structure with one set of alternating zonal jets is the final state by using a Lyapunov functional analysis. Since the evolution of the zonal band structure seen in their numerical experiment is similar to long-time behaviour of zonal jets on a rotating sphere mentioned above, we may deduce some physical insight about the stability of zonal flows induced by small-scale stochastic forcing by examining the system derived by Manfroi and Young more precisely.

Therefore in this paper, based on the work of Manfroi and Young [10], we investigate the stability of zonal jets with a background small-scale flow. $\S 2$ briefly introduces the model and the equation used in Manfroi and Young [10], and in $\S 3$, we derive a steady analytical solution of the Manfroi-Young equation. In $\S 4$, we investigate the linear stability of the steady solution both numerically and partially analytically, and also confirm its final state numerically. The discussions and the conclusions are given in the final section.

\section{The Manfroi-Young equation}

To investigate zonal flows induced by a small-scale deterministic forcing instead of a stochastic forcing, Manfroi and Young [10] derived the governing equation of a zonal flow having a small-scale sinusoidal transversal flow behind it on a $\beta$ plane, and studied their temporal evolution. Here, we briefly explain their derivation of the governing equation.

Let us consider an incompressible forced two-dimensional flow on a $\beta$ plane where the governing equation for such flow is written as $[11]^{1}$

$$
\frac{\partial Z}{\partial t}+J(\Psi, Z)+\beta \frac{\partial \Psi}{\partial x}=F+\nu \nabla^{2} Z
$$

\footnotetext{
${ }^{1}$ The vorticity equation in Vallis [11] takes into account of a large-scale drag, which will not be employed in this paper.
} 
Here $t, \Psi$, and $Z=\nabla^{2} \Psi$ are the time, the stream function, and the vorticity. $F$ and $\nu$ are the vorticity forcing function and the kinematic viscosity coefficient. $J(A, B)$ is the Jacobian operator: $J(A, B) \equiv(\partial A / \partial x)(\partial B / \partial y)-$ $(\partial A / \partial y)(\partial B / \partial x)$, and $\nabla=(\partial / \partial x, \partial / \partial y)$.

Suppose a steady base flow with the velocity

$$
\left(u_{B}, v_{B}\right)=\left(-\frac{\partial \Psi_{B}}{\partial y}, \frac{\partial \Psi_{B}}{\partial x}\right)=\left(U_{B}, m \Psi_{B 0} \sin m x\right)
$$

is driven by a suitable forcing function on this $\beta$ plane, where $\Psi_{B}, u_{B}$ and $v_{B}$ are the stream function, $x$ and $y$ components of the base flow, respectively, $U_{B}$ and $\Psi_{B 0}$ are constants, and $m$ is an integer. Now assume that the base flow is slightly unstable, that is, the Reynolds number of the base flow $R \equiv \Psi_{B 0} / \nu$ is slightly larger than the critical Reynolds number $R_{c}$,

$$
R=R_{c}\left(1+\epsilon^{2}\right)
$$

where $\epsilon$ is a small parameter. Then we write the total stream function of the flow as $\Psi=\Psi_{B}(x)+\psi(x, y, t)$, where $\psi(x, y, t)$ is the stream function of the disturbance flow and, from Eq. (1), satisfies

$$
\frac{\partial \zeta}{\partial t}+U_{B} \frac{\partial \zeta}{\partial x}+m \Psi_{B 0}\left(\frac{\partial \zeta}{\partial y}+m^{2} \frac{\partial \psi}{\partial y}\right) \sin (m x)+J(\psi, \zeta)+\beta \frac{\partial \psi}{\partial x}=\nu \nabla^{2} \zeta
$$

in which $\zeta \equiv \nabla^{2} \psi$ is the vorticity of the disturbance flow. Introducing new variables,

$$
(\hat{x}, \hat{y}) \equiv(m x, m y), \quad \hat{t} \equiv t m^{2} \nu, \quad \hat{\psi} \equiv \frac{\psi}{\nu},
$$

we nondimensionalize Eq. (3) as

$$
\frac{\partial \hat{\zeta}}{\partial \hat{t}}+\hat{u}_{B} \frac{\partial \hat{\zeta}}{\partial \hat{x}}+R\left(\frac{\partial \hat{\zeta}}{\partial \hat{y}}+\frac{\partial \hat{\psi}}{\partial \hat{y}}\right) \sin \hat{x}+J(\hat{\psi}, \hat{\zeta})+\hat{\beta} \frac{\partial \hat{\psi}}{\partial \hat{x}}=\hat{\nabla}^{2} \hat{\zeta}
$$

where

$$
\hat{u}_{B} \equiv \frac{u_{B}}{m \nu}, \quad \hat{\beta} \equiv \frac{\beta}{m^{3} \nu} .
$$

We, hereafter, drop the notation ` for the dimensionless variables and operators for simplicity. 
Using the small parameter $\epsilon$ defined in (2), we now perform perturbation expansions of the stream function of the disturbance flow $\psi, U_{B}$, and the $\beta$ parameter:

$$
\begin{aligned}
\psi & =\psi_{0}+\epsilon \psi_{1}+\epsilon^{2} \psi_{2}+\cdots, \\
U_{B} & =U_{B 0}+\epsilon U_{B 1}+\epsilon^{2} U_{B 2}+\cdots, \\
\beta & =\beta_{0}+\epsilon \beta_{1}+\epsilon^{2} \beta_{2}+\cdots,
\end{aligned}
$$

and multiple-scale expansions:

$$
\frac{\partial}{\partial x} \rightarrow \frac{\partial}{\partial x}+\epsilon^{6} \frac{\partial}{\partial \xi}, \quad \frac{\partial}{\partial y} \rightarrow \epsilon \frac{\partial}{\partial \eta}, \quad \frac{\partial}{\partial t} \rightarrow \epsilon^{4} \frac{\partial}{\partial \tau},
$$

where $\eta, \tau$ and $\xi$ are defined as

$$
\eta \equiv \epsilon y, \quad \tau \equiv \epsilon^{4} t, \quad \xi \equiv \epsilon^{6} x .
$$

Substituting the expanded variables and operators (5), (6), and (7) into Eq. (4), then we obtain the equation for the $O(1)$ elements

$$
\frac{\partial^{4} \psi_{0}}{\partial x^{4}}-U_{B 0} \frac{\partial^{3} \psi_{0}}{\partial x^{3}}-\beta_{0} \frac{\partial \psi_{0}}{\partial x}=0
$$

which is satisfied if $\psi_{0}$ depends on $\xi, \eta, \tau$ as

$$
\psi_{0}=A(\xi, \eta, \tau)
$$

Substituting the expanded variables and operators (5), (6), and (7) into Eq. (4) again, we next take the $x$-mean of it. By gathering the $O\left(\epsilon^{6}\right)$ elements, we obtain

$$
\begin{aligned}
\frac{\partial}{\partial \tau} \frac{\partial^{2} A}{\partial \eta^{2}}+2 \frac{\partial^{4} A}{\partial \eta^{4}}+3 \frac{\partial^{6} A}{\partial \eta^{6}}-\frac{\partial^{3}}{\partial \eta^{3}}[ & \left.\left(\beta_{1}-U_{B 1}+\frac{\partial A}{\partial \eta}\right)^{2} \frac{\partial A}{\partial \eta}\right] \\
& +\frac{1}{3} \frac{\partial^{3}}{\partial \eta^{3}}\left(\frac{\partial A}{\partial \eta}\right)^{3}+\beta_{0} \frac{\partial A}{\partial \xi}=0 .
\end{aligned}
$$

Considering the $\xi$-independent solution $A(\eta, \tau)$ of Eq. (8), Eq. (8) can be integrated over $\eta$ twice. After all, the governing equation for the $O(1)$ elements of the $x$-independent disturbance flow $U(\eta, \tau)$

$$
U(\eta, \tau) \equiv-\frac{\partial A(\eta, \tau)}{\partial \eta}
$$


is obtained as

$$
\frac{\partial U}{\partial \tau}=-\left(2-\gamma^{2}\right) \frac{\partial^{2} U}{\partial \eta^{2}}-3 \frac{\partial^{4} U}{\partial \eta^{4}}-2 \gamma \frac{\partial^{2} U^{2}}{\partial \eta^{2}}+\frac{2}{3} \frac{\partial^{2} U^{3}}{\partial \eta^{2}},
$$

where $\gamma$ is defined as

$$
\gamma \equiv \beta_{1}-U_{B 1}
$$

Eq. (9) governs zonal flows having a small-scale sinusoidal transversal background flow. We call Eq. (9) the Manfroi-Young equation, and investigate the linear stability of its steady solutions. The full derivation of Eq. (9) is available in $\S 4$ in Manfroi and Young [10].

The Manfroi-Young equation is a special form of the one-dimensional Cahn-Hilliard equation [3] whose steady solutions and their stabilities have attracted much attention. Langer [9] studied the linear stability of the onedimensional steady solution of the form of tanh, and also gave an intuitive explanation for the general stability criterion for a steady solution with a periodic boundary condition in an arbitrary dimensional case, which was extended to the cases of a natural boundary condition, or a null flux boundary condition, by Novick-Cohen and Segel [12]. Bettinson and Rowlands [2] performed linear stability analysis of a one-dimensional steady kink solution to a general Cahn-Hilliard equation in an infinite domain for both small- and large-wavenumber three-dimensional perturbations, and gave an approximate formula for eigenvalues by perturbation methods. The linear stability of a steady one-dimensional kink-antikink solution, which is called a bubble solution, and of a multibubble solution was studied by Argentina et al. [1]. The bifurcations originating from varying system size are discussed for both a periodic boundary condition and a natural boundary condition. VillainGuillot [19] suggested that steady non-symmetric soliton-lattice solutions, which belong to the family of the soliton-lattice solutions discussed in NovickCohen and Segel [12] are linearly unstable. Although a great deal of research has been carried out for the stability of steady solutions of the Cahn-Hilliard equation, to the best of the authors' knowledge, the steady solution which consists of one bump in an infinite domain and their linear stabilities relevant to the present $\beta$ plane problem have not been investigated yet. Thus in the following two sections, we derive steady solutions of the Manfroi-Young equation analytically and investigate their linear stability both numerically and analytically. 


\section{Analytical derivation of the steady solution}

Now, we consider a steady solution $U_{0}(\eta)$ of Eq. (9), having one bump and $U_{0} \rightarrow U_{W}$ as $\eta \rightarrow \pm \infty$, where $U_{W}$ is a constant ${ }^{2}$. The equation and boundary conditions for the $U_{0}$ are

$$
\begin{gathered}
-\left(2-\gamma^{2}\right) \frac{d^{2} U_{0}}{d \eta^{2}}-3 \frac{d^{4} U_{0}}{d \eta^{4}}-2 \gamma \frac{d^{2} U_{0}^{2}}{d \eta^{2}}+\frac{2}{3} \frac{d^{2} U_{0}^{3}}{d \eta^{2}}=0, \\
U_{0} \rightarrow U_{W}, \quad \frac{d U_{0}}{d \eta} \rightarrow 0 \quad \text { as } \eta \rightarrow \pm \infty .
\end{gathered}
$$

To solve Eq. (10a), we first integrate it over $\eta$ twice, and use conditions $(10 \mathrm{~b})^{3}$. Multiplying $d U_{0} / d \eta$ to both hand sides of the obtained equation, and integrating it with $\eta$ again, we have

$$
\frac{d U_{0}}{d \eta}= \pm \frac{1}{3} \sqrt{-V\left(U_{0}\right)}
$$

Here, the potential $V\left(U_{0}\right)$ is defined as

$$
V\left(U_{0}\right) \equiv-U_{0}^{4}+4 \gamma U_{0}^{3}+3\left(2-\gamma^{2}\right) U_{0}^{2}-6 C_{1} U_{0}-3 C_{2},
$$

and the constants of integration $C_{1}$ and $C_{2}$ are

$$
\begin{aligned}
& C_{1}=\left(2-\gamma^{2}\right) U_{W}+2 \gamma U_{W}^{2}-\frac{2}{3} U_{W}^{3}, \\
& C_{2}=U_{W}^{4}-\frac{8}{3} \gamma U_{W}^{3}-\left(2-\gamma^{2}\right) U_{W}^{2} .
\end{aligned}
$$

To realize the solution $U_{0}$ that takes the same value $U_{W}$ at $\eta \rightarrow \pm \infty$, the potential $V\left(U_{0}\right)$ should have the shape shown in Fig. 1, specifically; $V\left(U_{0}\right)$ must take a double root $U_{W}$ and two other different real roots $U_{E}$ and $U_{R}$ $\left(U_{E} \neq U_{R}\right)^{4} V\left(U_{0}\right)$, as a consequence, should be expressed as

$$
\begin{array}{r}
V\left(U_{0}\right)=\left(U_{0}-U_{W}\right)^{2}\left(U_{0}-U_{E}\right)\left(U_{0}-U_{R}\right), \\
U_{E}<U_{R}, \quad U_{W} \neq U_{E}, \quad U_{W} \neq U_{R} .
\end{array}
$$

\footnotetext{
${ }^{2}$ The steady solution having the eastward/westward bump is expressed as a steady solution with an/a eastward/westward jet hereafter.

${ }^{3}$ We adopt natural conditions $d^{2} U_{0} / d \eta^{2}, d^{3} U_{0} / d \eta^{3} \rightarrow 0(\eta \rightarrow \pm \infty)$

${ }^{4} U_{W}, U_{E}$ correspond to the $U_{W}, U_{E}$ in Fig. 3 in Manfroi and Young[10], and $U_{R}$ is the rest root. For the case $U_{0}$ is an eastward jet, $U_{W}$ and $U_{E}$ give the westward and eastward maximum values of the $U_{0}$, respectively; for the case $U_{0}$ is a westward jet, $U_{R}$ and $U_{W}$ give the westward and eastward maximum values of the $U_{0}$, respectively.
} 


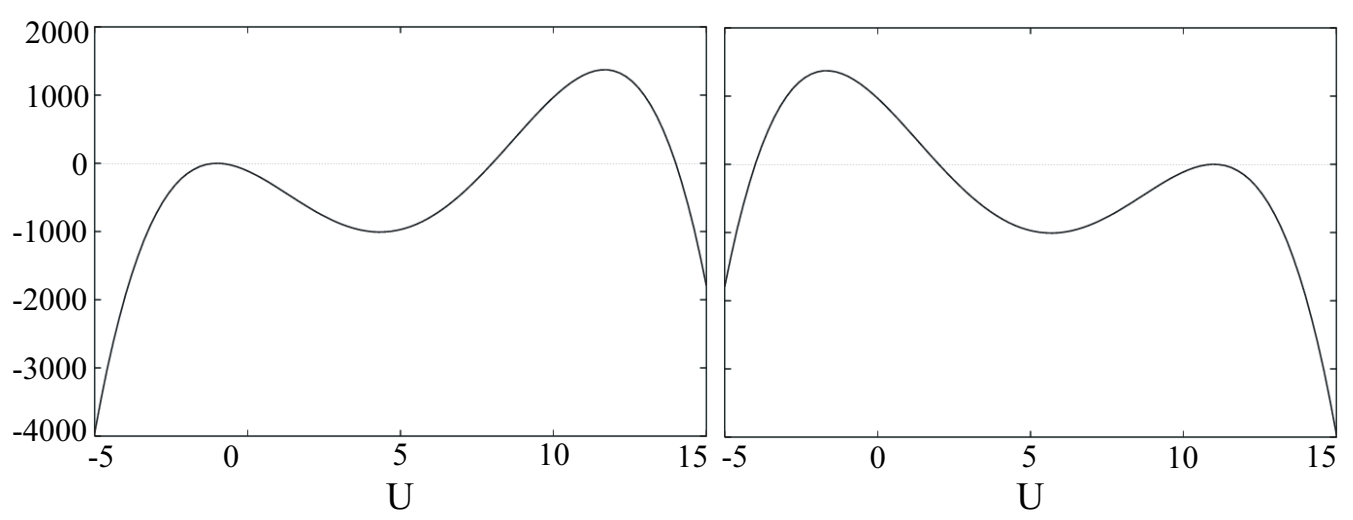

Figure 1: Examples of the shape of potential $V\left(U_{0}\right)$ which realizes a solution $U_{0}(\eta)$ that satisfies $U_{0} \rightarrow U_{W}$ as $\eta \rightarrow \pm \infty$. $U_{W}<U_{E}<U_{R}$ for $V\left(U_{0}\right)$ which realizes $U_{0}$ with an eastward jet (Left: for the case $\gamma=-5, U_{W}=1$ ), while $U_{E}<U_{R}<U_{W}$ for $V\left(U_{0}\right)$ which realizes $U_{0}$ with a westward jet (Right: for the case $\gamma=5, U_{W}=-1$ ).

From the definitions (12) and (13), on the other hand, $V\left(U_{0}\right)$ can be factorized as

$$
V\left(U_{0}\right)=-\left(U_{0}-U_{W}\right)^{2}\left\{U_{0}^{2}-2\left(2 \gamma-U_{W}\right) U_{0}-\left(6-3 \gamma^{2}+8 \gamma U_{W}-3 U_{W}^{2}\right)\right\} .
$$

As $V\left(U_{0}\right)$ has to be factorized to the form of Eq. (14), there must exist two different real roots $U_{E}$ and $U_{R}$ in addition to $U_{W}$. For the discriminant of the last factor of equation(15) to be positive, we have

$$
\gamma-\frac{1}{2} \sqrt{6\left(\gamma^{2}+2\right)}<U_{W}<\gamma+\frac{1}{2} \sqrt{6\left(\gamma^{2}+2\right)}
$$

Note that in the case where

$$
\begin{aligned}
U_{W}=\gamma-\frac{1}{2} \sqrt{6\left(\gamma^{2}+2\right)} \\
\text { or } \\
U_{W}=\gamma+\frac{1}{2} \sqrt{6\left(\gamma^{2}+2\right)}
\end{aligned}
$$

$V\left(U_{0}\right)$ has two real double roots $U_{W}$ and $U_{E}=U_{R}$, the only solution of (10a) and (10b) is $U_{0}=U_{W}{ }^{5}$. The parameters $U_{W}, U_{E}$, and $U_{R}$ should satisfy

\footnotetext{
${ }^{5}$ Apart from this uniform solution, if we do not assume that $U_{0}$ should converge to a
} 
$U_{W}<U_{E}<U_{R}$ (for an eastward jet) or $U_{E}<U_{R}<U_{W}$ (for a westward jet) as shown in Fig. 1. Therefore, the last factor of (15),

$$
U_{0}^{2}-2\left(2 \gamma-U_{W}\right) U_{0}-\left(6-3 \gamma^{2}+8 \gamma U_{W}-3 U_{W}^{2}\right)=\left(U_{0}-U_{E}\right)\left(U_{0}-U_{R}\right),
$$

has to be positive at $U_{0}=U_{W}$, and thus

$$
U_{W}<\gamma-\frac{1}{2} \sqrt{2\left(\gamma^{2}+2\right)} \quad \text { or } \quad \gamma+\frac{1}{2} \sqrt{2\left(\gamma^{2}+2\right)}<U_{W}
$$

should be satisfied. Again note that the case

$$
\begin{aligned}
U_{W}=\gamma-\frac{1}{2} \sqrt{2\left(\gamma^{2}+2\right)} \\
\text { or } \\
U_{W}=\gamma+\frac{1}{2} \sqrt{2\left(\gamma^{2}+2\right)} .
\end{aligned}
$$

corresponds to the situation that either $U_{E}$ or $U_{R}$ is the same value as the double root $U_{W}$.

The conditions (16) and (18) are shown in Fig. 2 where the hatched regions give $\left(\gamma, U_{W}\right)$ corresponding to a steady solution $U_{0}$ which has one bump and takes the value $U_{W}$ at $\eta \rightarrow \pm \infty$. The upper hatched region corresponds to $U_{0}$ with a westward jet, while lower hatched region to $U_{0}$ with an eastward jet.

Under the conditions of (16) and (18), $U_{0}$ has a double real root $U_{W}$ and two other different real roots $U_{E}$ and $U_{R}$;

$$
\begin{aligned}
& U_{E}=2 \gamma-U_{W}-\sqrt{-2 U_{W}^{2}+4 \gamma U_{W}+\gamma^{2}+6}, \\
& U_{R}=2 \gamma-U_{W}+\sqrt{-2 U_{W}^{2}+4 \gamma U_{W}+\gamma^{2}+6} .
\end{aligned}
$$

same value, $U_{W}$, as $\eta$ goes to $\pm \infty$, Eq. (10a) has a sigmoid solution

$$
U_{0 s i g}= \pm\left(U_{E}+\frac{U_{W}-U_{E}}{\exp \left[(\eta-c)\left(U_{W}-U_{E}\right) / 3\right]+1}\right),
$$

where $c$ is a constant which determine the center position of these solutions. These solution $U_{0 s i g}$ are the asymptotic forms of the rising and decreasing curves of $U_{0}$, i.e. $U_{0}$ is made by superposing two $U_{0 s i g}$ of different signs. 


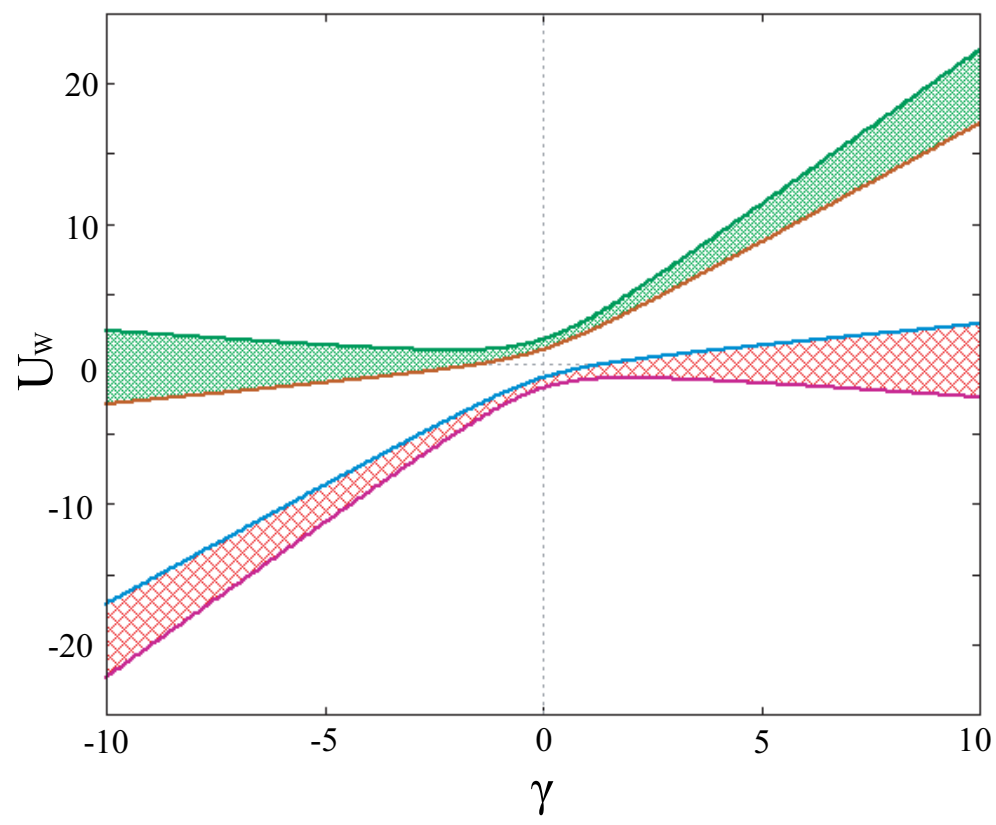

Figure 2: The regions of $\left(\gamma, U_{W}\right)$ which realize one-bump steady solution $U_{0}$. The upper and lower hatched regions correspond to $U_{0}$ with an eastward jet and with a westward jet, respectively. The boundary curves are, from above, (17b)(dark green), (19b)(brown), (19a)(light blue), and (17a)(purple).

Eq. (11) is then written as

$$
\frac{d U_{0}}{d \eta}= \pm \frac{1}{3} \sqrt{\left(U_{0}-U_{W}\right)^{2}\left(U_{0}-U_{E}\right)\left(U_{0}-U_{R}\right)}
$$

A steady solution with an eastward jet $U_{0 e a s t}(\eta)\left(U_{W} \leq U_{0 e a s t}(\eta) \leq U_{E}\right)$ is obtained by integrating the equation (22) as

$$
\int_{\eta_{E}}^{\eta} d \eta= \pm 3 \int_{U_{E}}^{U_{0 e a s t}(\eta)} \frac{d U_{0}}{\left(U_{0}-U_{W}\right) \sqrt{\left(U_{0}-U_{E}\right)\left(U_{0}-U_{R}\right)}}
$$




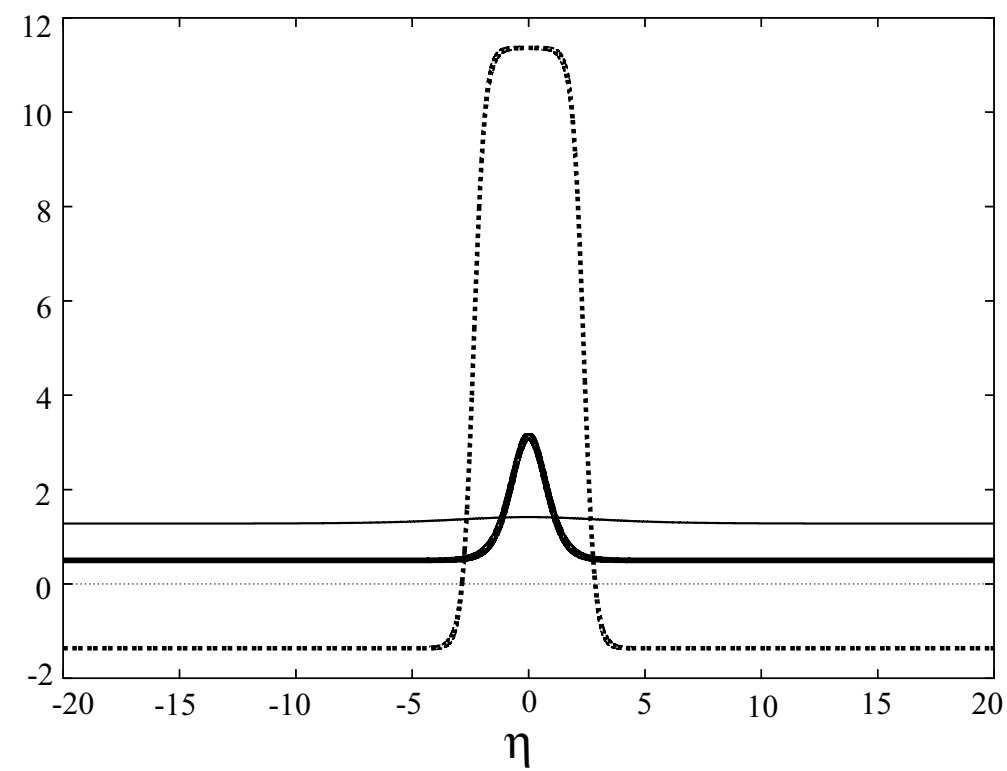

Figure 3: Examples of steady solutions with an eastward jets; $\left(\gamma, U_{W}\right)=$ $(5.0,-1.363961)$ :dashed line, $(5.0,0.5)$ :thicker solid line, $(5.0,1.28)$ :thinner solid line.

where $\eta_{E}$ is defined to satisfy $U_{\text {0east }}\left(\eta_{E}\right)=U_{E}$, which leads to

$$
\begin{aligned}
U_{\text {0east }}(\eta) & =\frac{a_{\text {east }}^{2} U_{R} \tanh ^{2}\left[\frac{\left(U_{R}-U_{W}\right) a_{\text {east }}}{6} \eta\right]-U_{E}}{a_{\text {east }}^{2} \tanh ^{2}\left[\frac{\left(U_{R}-U_{W}\right) a_{\text {east }}}{6} \eta\right]-1}, \\
a_{\text {east }} & \equiv \sqrt{\frac{U_{E}-U_{W}}{U_{R}-U_{W}}} .
\end{aligned}
$$

Examples of $U_{\text {Deast }}(\eta)$ are shown in Fig. 3 for some combinations of $\gamma$ and $U_{W}$. Jets near the boundary curve (17a) have trapezoid-like shape; jets near the boundary curve (19a) are almost flat; and jets apart from the boundaries have sharp shapes. A steady solution with a westward jet $U_{0 \text { west }}(\eta)\left(U_{R} \leq\right.$ 
$\left.U_{0 w e s t}(\eta) \leq U_{W}\right)$ is similarly obtained as

$$
\begin{aligned}
U_{0 \text { west }}(\eta) & =\frac{a_{\text {west }}^{2} U_{E} \tanh ^{2}\left[\frac{\left(U_{E}-U_{W}\right) a_{\text {west }}}{6} \eta\right]-U_{R}}{a_{\text {west }}^{2} \tanh ^{2}\left[\frac{\left(U_{E}-U_{W}\right) a_{\text {west }}}{6} \eta\right]-1}, \\
a_{\text {west }} & \equiv \sqrt{\frac{U_{R}-U_{W}}{U_{E}-U_{W}}} .
\end{aligned}
$$

Note that no steady solution exists on the boundary curves (17a), (17b), (19a), and (19b).

\section{Linear stability of steady isolated jets}

\subsection{Characteristic equation and eigenvalues}

To investigate the linear stability of a steady solution $U_{0}(\eta)$, we first derive its characteristic equation. Consider the case in which a sufficiently small perturbation $v(\eta, \tau)$ is added to the steady solution $U_{0}(\eta)$. Substituting $U=$ $U_{0}+v$ for $U$ in Eq. (9), we linearize it with respect to $v$. Further, we assume $v$ to be in the following form,

$$
v=\exp (\sigma \tau) f(\eta)
$$

where $f(\eta)$ is a certain function, which satisfies,

$$
f \rightarrow 0, \quad \frac{d f}{d \eta} \rightarrow 0 \quad \text { as } \eta \rightarrow \pm \infty .
$$

Then, we have

$$
\sigma f=\frac{d^{2}}{d \eta^{2}}\left\{\left[-\left(2-\gamma^{2}\right)+\left(2 U_{0}^{2}-4 \gamma U_{0}\right)\right] f-3 \frac{d^{2} f}{d \eta^{2}}\right\} .
$$

Assume that $\sigma \neq 0$. By integrating Eq. (25) over $\eta$ twice, we find

$$
\int_{-\infty}^{\infty} \int_{-\infty}^{\eta} f\left(\eta^{\prime}\right) d \eta^{\prime} d \eta=0,{ }^{6}
$$

\footnotetext{
${ }^{6}$ We assume $d^{2} f / d \eta^{2}, d^{3} f / d \eta^{3} \rightarrow 0(\eta \rightarrow \pm \infty)$ as in equation(10a)
} 
Now rewriting $f(\eta)$ as

$$
f(\eta)=\frac{d^{2} g(\eta)}{d \eta^{2}}, \quad g(\eta) \rightarrow 0, \quad \frac{d g(\eta)}{d \eta} \rightarrow 0, \quad \text { as } \eta \rightarrow-\infty .
$$

We have

$g(\eta)=g(\eta)-g(-\infty)=\int_{-\infty}^{\eta} \int_{-\infty}^{\eta^{\prime}} \frac{d^{2} g\left(\eta^{\prime \prime}\right)}{d \eta^{\prime \prime 2}} d \eta^{\prime \prime} d \eta^{\prime}=\int_{-\infty}^{\eta} \int_{-\infty}^{\eta^{\prime}} f\left(\eta^{\prime \prime}\right) d \eta^{\prime \prime} d \eta^{\prime}$.

This means that, $g, d g / d \eta \rightarrow 0$ as $\eta \rightarrow \pm \infty$. Putting $f=d^{2} g / d \eta^{2}$ into Eq. (25), and integrating this equation over $\eta$ twice, the characteristic equation is obtained as follows.

$$
\begin{gathered}
\sigma g=\left[-\left(2-\gamma^{2}\right)+\left(2 U_{0}^{2}-4 \gamma U_{0}\right)\right] \frac{d^{2} g}{d \eta^{2}}-3 \frac{d^{4} g}{d \eta^{4}}, \\
g \rightarrow 0, \quad \frac{d g}{d \eta} \rightarrow 0 \quad \text { as } \eta \rightarrow \pm \infty
\end{gathered}
$$

From the symmetry property of the characteristic equation, it easily follows that investigating the linear stability of $U_{\text {0east }}$ with $\gamma \geq 0$ is enough to know the linear stability of all the $U_{0}$ (Appendix). It is easily verified that the characteristic equation (26) also holds for $\sigma=0$.

We solve eigenvalue problem (26) numerically by the Fourier spectral method, where $U_{0}, U_{0}^{2}, g$ are expressed as

$$
\begin{aligned}
U_{0} & =\sum_{k=-K}^{K} u_{k} \exp \left(i k \frac{2 \pi}{L} \eta\right), \\
U_{0}^{2} & =\sum_{k=-K}^{K} d_{k} \exp \left(i k \frac{2 \pi}{L} \eta\right), \\
g & =\sum_{k=-K}^{K} c_{k} \exp \left(i k \frac{2 \pi}{L} \eta\right) .
\end{aligned}
$$

We consider the domain $[0, L]$ and a periodic boundary condition for $\eta$. The width of the domain, $L$, was determined so that the numerical calculation converges sufficiently (We have performed numerical calculations with both $L=150$ and $L=225$, and have confirmed that the relative errors are less 
than $0.1 \%$.). The width of the spatial grids was set to be around $10^{-3}$, and the number of modes $K$ was taken in the way that the maximum wavenumber $2 \pi K / L$ becomes $10 \pi$ for each case.

Fig. 4 shows the real parts of the first eigenvalues, $\sigma_{f r} \equiv \operatorname{Max}\left\{\operatorname{Re}(\sigma) \mid\left(\gamma, U_{W}\right)\right.$ : fixed \}, for $\gamma=0.0,1.0$, and 5.0 and $U_{W}$ satisfying the conditions (16) and (18). It is apparent that every $\sigma_{f r}$ is positive and that the maximum value of $\sigma_{f r}$ becomes larger for the larger $\gamma$, while $\sigma_{f r}$ converges to 0 as $U_{W}$ approaches the two boundary curves (17a) and (19a) (the purple and the light blue curves in Figs. 2 and 4). The square roots of $\sigma_{f r}$ are plotted in Fig. 5. It is clear that $\sqrt{\sigma_{f r}}$ are aligned in the vicinity of the point on the two boundaries (17a) and (19a), and the zeros on the line obtained by the least square fitting are quite precisely on the boundaries (17a) and (19a) with the relative errors less than $0.4 \%$ for $\gamma=0,1.0$, and 5.0. The numerical results therefore show that the real part of every first eigenvalue is positive, implying that the steady solutions $U_{0}\left(\eta ; \gamma, U_{W}\right)$ are all linearly unstable.

\subsection{The analytical evaluation of the eigenvalues}

It is numerically showed in $\S 4.1$ that, for a fixed $\gamma$, the real part of the first eigenvalues $\sigma_{f r}=\operatorname{Max}\left\{\operatorname{Re}(\sigma) \mid\left(\gamma, U_{W}\right)\right.$ : fixed $\}$ has zeros of the second order with respect to $U_{W}$ on the curves (17a) and (19a). This feature is partly confirmed analytically below.

\subsubsection{Around the upper boundary}

On the boundary of the curve (19a), $U_{W}$ is given by

$$
U_{W}=\gamma-\frac{1}{2} \sqrt{2\left(\gamma^{2}+2\right)} \equiv U_{W_{C}}
$$

which give $U_{E} \equiv U_{E_{C}}=U_{W_{C}}$ and

$$
U_{R}=\left(2 \gamma-U_{W_{C}}\right)+\sqrt{-2 U_{W_{C}}^{2}+4 \gamma U_{W_{C}}+\gamma^{2}+6} \equiv U_{R_{C}},
$$

and then

$$
U_{E_{C}}-U_{W_{C}}=2\left(\gamma-U_{W_{C}}\right)-\sqrt{-2 U_{W_{C}}^{2}+4 \gamma U_{W_{C}}+\gamma^{2}+6}=0 .
$$

Let us consider a point close to $C\left(\gamma, U_{W_{C}}\right): N\left(\gamma, U_{W}=U_{W_{C}}-\delta\right)$, where $\delta$ is positive and small. As $U_{W_{C}}=U_{E_{C}}$ on the curve (19a), $U_{E}$ at the point $N$ is 
expressed as $U_{E}=U_{W}+\epsilon$, where

$$
\begin{aligned}
\epsilon & \equiv U_{E}-U_{W} \\
& =2\left(\gamma-U_{W}\right)-\sqrt{-2 U_{W}^{2}+4 \gamma U_{W}+\gamma^{2}+6} \\
& =2\left(\gamma-U_{W_{C}}+\delta\right)-\sqrt{-2\left(U_{W_{C}}-\delta\right)^{2}+4 \gamma\left(U_{W_{C}}-\delta\right)+\gamma^{2}+6} .
\end{aligned}
$$

Using Eqs. (27) and (29), $\epsilon$ is given as

$$
\epsilon=3 \delta+\mathrm{O}\left(\delta^{2}\right) .
$$

From (28) and (20), we have

$$
2 U_{W}^{2}-4 \gamma U_{W}-\left(2-\gamma^{2}\right)=\frac{4}{3}\left(\gamma-U_{W}\right) \epsilon+\mathrm{O}\left(\epsilon^{2}\right) .
$$

Noting that $a^{2}=\mathrm{O}(\sqrt{\epsilon})$, the steady solution $U_{0}$ on the point $N$ can be written from (23a) as

$$
U_{0} \equiv U_{W}+\epsilon S(\sqrt{\epsilon} \eta)+O\left(\epsilon^{2}\right)
$$

where

$$
S(\sqrt{\epsilon} \eta)=\operatorname{sech}^{2}\left[\frac{\sqrt{U_{R}-U_{W}}}{6} \sqrt{\epsilon} \eta\right] .
$$

The characteristic equation (26) precise to $\mathrm{O}(\epsilon)$ is, therefore,

$$
\sigma g=\left[\left(\gamma-U_{W}\right)\left(\frac{4}{3}-4 S(\sqrt{\epsilon} \eta)\right) \epsilon\right] \frac{d^{2} g}{d \eta^{2}}-3 \frac{d^{4} g}{d \eta^{4}} .
$$

Here, Eq. (31) has been used. Defining $s \equiv \sqrt{\epsilon} \eta$, Eq. (32) becomes

$$
\sigma_{1} g=\left[\left(\gamma-U_{W}\right)\left(\frac{4}{3}-4 S(s)\right)\right] \frac{d^{2} g}{d s^{2}}-3 \frac{d^{4} g}{d s^{4}} .
$$

where $\sigma_{1}=\sigma / \epsilon^{2}$. Hence, an eigenvalue $\sigma$ given by Eq. (32) can be expressed with an eigenvalue $\sigma_{1}$ given by Eq. (33) as

$$
\sigma=\epsilon^{2} \sigma_{1}=9 \sigma_{1} \delta^{2}=9 \sigma_{1}\left(U_{W_{C}}-U_{W}\right)^{2} .
$$

where (30) has been used. Eq. (34) demonstrates that the eigenvalues in the vicinity of the point $C\left(\gamma, U_{W_{C}}\right)$ behave as $9 \sigma_{1}\left(U_{W_{C}}-U_{W}\right)^{2}$.

Fig. 6 shows the real parts of the first eigenvalues $\sigma_{f r}$, the two zeros obtained from the fitted line in $\S 4.1$, and the analytical curve (34), for $\gamma=$ $0.0,1.0$, and 5.0 and $U_{W}$ which satisfy the conditions (16) and (18). $\sigma_{1}$ is obtained as $\sigma_{1}=0.111111,0.166668$, and 1.500000 , respectively, by solving (33) numerically. The analytical curve fits fairly well with the first eigenvalues in the vicinity of the point $C\left(\gamma, U_{W_{C}}\right)$. 


\subsubsection{Around the lower boundary}

For the eigenvalues at $\left(\gamma, U_{W}\right)$ close to the other boundary curve (17a), we analytically show below that the eigenvalues converge to zero as $\left(\gamma, U_{W}\right)$ approaches an arbitrary point $D$ on the boundary curve (17a).

Let us denote $U_{W}$ at $D$ as $U_{W_{D}}$,

$$
U_{W_{D}}=\gamma-\frac{1}{2} \sqrt{6\left(\gamma^{2}+2\right)},
$$

and consider a point $M$ close to the point $D\left(\gamma, U_{W_{D}}\right): M\left(\gamma, U_{W}=U_{W_{D}}+\delta\right)$, where $\delta$ is positive and small. Then $a$ defined in Eq. (23b) at $M$ satisfies

$$
a^{2}=1-\epsilon+\mathrm{O}(\delta)
$$

where $\epsilon$ is defined as

$$
\epsilon \equiv \frac{2 \sqrt{2 \sqrt{6\left(\gamma^{2}+2\right)} \delta-2 \delta^{2}}}{\sqrt{6\left(\gamma^{2}+2\right)}} .
$$

Introducing $\alpha$ by

$$
\begin{aligned}
\alpha & \equiv \frac{a}{6}\left(U_{R}-U_{W}\right) \\
& =\frac{\sqrt{6\left(\gamma^{2}+2\right)}}{6}\left(1-\frac{\epsilon}{2}+\mathrm{O}(\delta)\right),
\end{aligned}
$$

we can write $U_{0}(\eta)$ as

$$
U_{0}(\eta)=\gamma-\delta+\frac{1}{2} \sqrt{6\left(\gamma^{2}+2\right)}(1+\epsilon)-\frac{\sqrt{6\left(\gamma^{2}+2\right)}}{\operatorname{sech}^{2}(\alpha \eta) / \epsilon+\tanh ^{2}(\alpha \eta)+\mathrm{O}(\delta)}
$$

for small $\delta$. Now write $\eta$ as $\eta=\eta_{0}+\eta^{\prime}$, where $\eta_{0}$ is defined such that

$$
\operatorname{sech}\left(\alpha \eta_{0}\right)=\sqrt{\epsilon},
$$

then, when $\delta \rightarrow 0$,

$$
\frac{d U_{0}\left(\eta_{0}+\eta^{\prime}\right)}{d \eta^{\prime}}=-\frac{1}{6}\left(-2 U_{0}^{2}+4 \gamma U_{0}+\gamma^{2}+6\right),
$$


and after all, the characteristic equation (26) becomes

$$
\sigma g=\left[6 \frac{d U_{0}}{d \eta^{\prime}}+2\left(2+\gamma^{2}\right)\right] \frac{d^{2} g}{d \eta^{\prime 2}}-3 \frac{d^{4} g}{d \eta^{\prime 4}}
$$

Multiplying $d^{2} g^{\dagger} / d \eta^{\prime 2}$, where $g^{\dagger}$ is an adjoint solution of $g$, to both sides of Eq. (35) and integrating this over $\eta$, we obtain

$$
-\sigma \int\left|\frac{d g}{d \eta^{\prime}}\right|^{2} d \eta^{\prime}=\int\left[6 \frac{d U_{0}}{d \eta^{\prime}}+2\left(2+\gamma^{2}\right)\right]\left|\frac{d^{2} g}{d \eta^{\prime 2}}\right|^{2} d \eta^{\prime}+3 \int\left|\frac{d^{3} g}{d \eta^{\prime 3}}\right|^{2} d \eta^{\prime} .
$$

We next introduce $\phi \equiv d^{2} g / d \eta^{2}$, and define a function

$$
\begin{aligned}
I[\phi] & \equiv \int\left[6 \frac{d U_{0}}{d \eta^{\prime}}|\phi|^{2}+3\left|\frac{d \phi}{d \eta^{\prime}}\right|^{2}\right] d \eta^{\prime} \\
& =\int\left[-3\left(\gamma^{2}+2\right) \operatorname{sech}^{2}\left(\alpha \eta^{\prime}\right)|\phi|^{2}+3\left|\frac{d \phi}{d \eta^{\prime}}\right|^{2}\right] d \eta^{\prime} .
\end{aligned}
$$

Now, we know that the Schrödinger equation

$$
\left(-\frac{\hbar^{2}}{2 m} \frac{d^{2}}{d x^{2}}-A_{0} \operatorname{sech}^{2} \beta x\right) \psi=E \psi, \quad A_{0}>0,
$$

has its minimum eigenvalue (Landau-Lifshitz [8]),

$$
E_{0}=-\frac{\hbar^{2} \beta^{2}}{8 m}\left[-1+\sqrt{1+\frac{8 m A_{0}}{\hbar^{2} \beta^{2}}}\right]^{2}
$$

and therefore, we have

$$
\int\left(\frac{\hbar^{2}}{2 m}\left|\frac{d \psi}{d x}\right|^{2}-A_{0} \operatorname{sech}^{2} \beta x|\psi|^{2}\right) d x \geq E_{0} \int|\psi|^{2} d x .
$$

Applying this to Eq. (37) yields

$$
I[\phi] \geq-2\left(\gamma^{2}+2\right) \int|\phi|^{2} d \eta^{\prime},
$$

hence, from Eq. (36),

$$
-\sigma \int\left|\frac{d g}{d \eta^{\prime}}\right|^{2} d \eta^{\prime}=I[\phi]+2\left(\gamma^{2}+2\right) \int|\phi|^{2} d \eta^{\prime} \geq 0 ;
$$

this certifies the limit of the eigenvalue $\sigma$ as $\delta \rightarrow 0$ is real and non-positive, which, together with Fig. 4 and Fig. 5, indicates $\lim _{\delta \rightarrow 0} \sigma=0$ at the point $D$. 


\subsection{The growth of an unstable eigenfunction and the final flow field}

As discussed above, every non-trivial steady solution $U_{0}(\eta)$ of Eq. (9) is linearly unstable. The next question is the final state $($ at $\tau=\infty$ ) of this unstable jet when it is slightly perturbed.

Fig. 7 shows the first eigenfunction and the time development of $U_{0}$ added its first eigenfunction as the perturbation for the case $\left(\gamma, U_{W}\right)=(1.0,-1.05)$. The amplitude of the perturbation function is set to be $3 \%$ of that of $U_{0}$. The solution of Eq. (9) is numerically calculated with the Fourier spectral method. The time integration is performed with the 4th order Runge-Kutta method with a time step width $\Delta \tau=1.0 \times 10^{-5}$. The boundary condition, the width of the domain, the number of the mode $K$ for the Fourier expansion, and the number of the grid point are all set to be the same with those used for the calculation for the eigenvalues in $\S 4$. In Fig. 7 , the jet become flat gradually, and the uniform flow is realised at the end. Note that it is easy to verify that this uniform flow $U=U_{W}$ is linearly stable in the ranges shown in Fig. $2^{7}$.

\section{Discussions and Conclusions}

In this paper, we have considered zonal jet flows on a $\beta$ plane subject to the effect of a background small-scale non-zonal flow. Using the evolution equation developed by Manfroi and Young [10], analytical solutions for steady isolated jets have been obtained. The linear stability of the steady jets has been investigated both numerically and partially analytically, and it is found that the steady jet solutions are all linearly unstable. Numerical time integrations of the evolution equation also showed that the perturbed unstable steady jet solution gradually decreases its amplitude, and becomes a uniform flow in the long run.

Comparing the results shown in this paper to the numerical experiment performed by Manfroi and Young [10], there seems to be a disagreement

\footnotetext{
${ }^{7} U=U_{W}$ is linearly stable if$$
U_{W} \leq \gamma-\frac{1}{2} \sqrt{2\left(\gamma^{2}+2\right)} \quad \text { or } \quad \gamma+\frac{1}{2} \sqrt{2\left(\gamma^{2}+2\right)} \leq U_{W} .
$$ 
at first sight. Every steady jet solution is linearly unstable, whilst the jets appear in the numerical simulation in Manfroi and Young [10] disappear one by one quite deliberatively, ending up with one jet in the considering domain at the final stage.

This disagreement may be caused by the fact that the first eigenvalues have zeros of second order on the curves (17a), (17b), (19a), and (19b). In Manfroi and Young [10], they only fixed $\gamma$ in advance in their numerical simulation, where $U_{W}$ was "selected" spontaneously by the system itself and the resultant parameters $\left(\gamma, U_{W}\right)$ was very close to the boundary curve (17a), which suggests that the zonal jets in the simulation are almost marginally stable. This implies that although the jets seen in the numerical simulation in Manfroi and Young [10] really were linearly unstable, their instability was very weak and the jets have behaved as if they had been linearly stable.

Concerning the difference between the analyses in Manfroi and Young [10] and our study, we also have to mention the differences of the boundary condition. The solution in Manfroi and Young [10] is affected by the periodic boundary condition, and alternative eastward westward jets rather than an isolated jet are observed. We, on the other hand, perform the analyses in the infinitely extended domain. For the calculations of the eigenvalue problems and numerical time integrations, we take a sufficiently large computational domain in order that the amplitude of disturbances may vanish when it is far from the isolated jets. Note that the eigenfunction decays spatially slower than the jet. The periodic boundary condition allows the baseline shift of the jet profile, which does not occur when the equation is considered in an infinite domain. Therefore, strictly speaking, the result of our analyses cannot be applied to the final state of their solution.

Nevertheless, we may find a point of our result consistent with the numerical experiment by Manfroi and Young [10]. Disappearance of the thin jet seen in Fig. 3 in Manfroi and Young [10] at $\tau=500-600$ may be caused by the intrinsic instability of the jet obtained in the present study, firstly because the baseline of the jet does not vary during the disappearance of the jet, consistently with the presumption of our analysis, and secondary because the stability of the thin jet is considered not to be affected by the adjacent jets, as the eigenfunction of the unstable disturbance is concentrated around the basic jet (Fig. 7).

The applicability of the results of the present study to the merger/disappearance of the zonal jets in the problems of stochastically forced two-dimensional turbulence on a $\beta$ plane or a rotating sphere (Chekhlov et al. [4], Obuse et al. 
[14]) is not clear, because the background turbulence in the case in Chekhlov et al. [4] and Obuse et al. [14] consists of a lot of wavy modes varying in time, and the background single sinusoidal flow adopted in this study may be too simple to incorporate the effect of turbulence. However, the notion of the instability of a jet caused by the background flow appears to work in the complex flow at least phenomenologically, and deserves further investigation.

\section{Appendix: The symmetry of the characteristic equation (26)}

Here, we show that it is sufficient to investigate $U_{\text {eeast }}$ with $\gamma \geq 0$ to know the linear stability of all steady solutions $U_{0}(\eta)$. For the sake of convenience, we divide the parameter region which realizes $U_{0}$ into four sectors:

$$
\begin{aligned}
& \mathrm{R}_{\text {east }} \equiv\left\{\left(\gamma, U_{W}\right) \mid \gamma-2 \sqrt{6\left(\gamma^{2}+2\right)}<U_{W}<\gamma-\frac{1}{2} \sqrt{2\left(\gamma^{2}+2\right)}, \gamma \geq 0\right\}, \\
& \mathrm{R}_{\text {east- }} \equiv\left\{\left(\gamma, U_{W}\right) \mid \gamma-2 \sqrt{6\left(\gamma^{2}+2\right)}<U_{W}<\gamma-\frac{1}{2} \sqrt{2\left(\gamma^{2}+2\right)}, \gamma \leq 0\right\}, \\
& \mathrm{R}_{\text {west }+} \equiv\left\{\left(\gamma, U_{W}\right) \mid \gamma+2 \sqrt{6\left(\gamma^{2}+2\right)}>U_{W}>\gamma+\frac{1}{2} \sqrt{2\left(\gamma^{2}+2\right)}, \gamma \geq 0\right\}, \\
& \mathrm{R}_{\text {west- }} \equiv\left\{\left(\gamma, U_{W} \mid \gamma+2 \sqrt{6\left(\gamma^{2}+2\right)}>U_{W}>\gamma+\frac{1}{2} \sqrt{2\left(\gamma^{2}+2\right)}, \gamma \leq 0\right\} .\right.
\end{aligned}
$$

$\mathrm{R}_{\text {east }+}, \mathrm{R}_{\text {east- }}, \mathrm{R}_{\text {west }+}$, and $\mathrm{R}_{\text {west- }}$ correspond to $U_{\text {0east }}$ with $\gamma \geq 0, U_{\text {0east }}$ with $\gamma \leq 0, U_{0 \text { west }}$ with $\gamma \geq 0$, and $U_{0 \text { west }}$ with $\gamma \leq 0$ respectively.

Now, take an arbitrary combination $\left(\gamma, U_{W}\right) \in \mathrm{R}_{\text {east }+}$, and consider $\left(-\gamma, U_{W}-\right.$ $2 \gamma$ ), which is in the sector $R_{\text {east- }}$. Then, from the definitions (20), (21), (23b), and $(23 \mathrm{a})$, the relations

$$
\begin{aligned}
U_{\text {Eeast }}^{-} & =U_{\text {Eeast }}^{+}-2 \gamma, \\
U_{\text {Reast }}^{-} & =U_{\text {Reast }}^{+}-2 \gamma, \\
a_{\text {east }}^{-} & =a_{\text {east }}^{+}, \\
U_{\text {0east }}^{-} & =U_{\text {0east }}^{+}-2 \gamma,
\end{aligned}
$$

hold. Subscripts east,${ }^{+}$and ${ }^{-}$above represent eastward jet, $\mathrm{R}_{\text {east+ }}$, and $\mathrm{R}_{\text {east- }}$ respectively; for example, $U_{\text {Eeast }}^{-}$means $U_{E}$ at $\left(-\gamma, U_{W}-2 \gamma\right) \in R_{\text {east-. }}$. Using 
Eq. (40), the characteristic equation (26) for $U_{0 e a s t}^{-}$can be written as

$$
\begin{aligned}
\sigma g & =\left[-\left(2-\gamma^{2}\right)+\left(2\left(U_{\text {0east }}^{-}\right)^{2}-4 \gamma U_{\text {0east }}^{-}\right)\right] \frac{d^{2} g}{d \eta^{2}}-3 \frac{d^{4} g}{d \eta^{4}} \\
& =\left[-\left(2-\gamma^{2}\right)+\left(2\left(U_{\text {0east }}^{+}\right)^{2}-4 \gamma U_{\text {0east }}^{+}\right)\right] \frac{d^{2} g}{d \eta^{2}}-3 \frac{d^{4} g}{d \eta^{4}}
\end{aligned}
$$

which is the same characteristic equation for $U_{\text {0east }}^{+}$. Hence, investigating the stability of $U_{0 \text { east }}^{+}$will certainly tell the stability of $U_{\text {0east }}$.

Next, take an arbitrary combination $\left(\gamma, U_{W}\right) \in \mathrm{R}_{\text {east+ }}$ again, and consider $\left(-\gamma,-U_{W}\right)$, which is easily show to be in the sector $R_{\text {west-. }}$. Then, from the definitions (20), (21), (23b), and (23a), the relations

$$
\begin{aligned}
U_{\text {Ewest }}^{-} & =-U_{\text {Reast }}^{+}, \\
U_{\text {Rwest }}^{-} & =-U_{\text {Eeast }}^{+}, \\
a_{\text {west }}^{-} & =a_{\text {east }}^{+}, \\
U_{\text {0west }}^{-} & =-U_{\text {0east }}^{+}
\end{aligned}
$$

hold. Using Eq. (41), the characteristic Eq. (26) for $U_{0 w e s t}^{-}$appears to be written as

$$
\begin{aligned}
\sigma g & =\left[-\left(2-\gamma^{2}\right)+\left(2\left(U_{\text {0west }}^{-}\right)^{2}-4 \gamma U_{0 \text { west }}^{-}\right)\right] \frac{d^{2} g}{d \eta^{2}}-3 \frac{d^{4} g}{d \eta^{4}} \\
& =\left[-\left(2-\gamma^{2}\right)+\left(2\left(U_{\text {0east }}^{+}\right)^{2}-4 \gamma U_{\text {0east }}^{+}\right)\right] \frac{d^{2} g}{d \eta^{2}}-3 \frac{d^{4} g}{d \eta^{4}}
\end{aligned}
$$

which is the same characteristic equation for $U_{\text {0east }}^{+}$. Hence, investigating the stability of $U_{0 \text { east }}^{+}$will also certainly tell the stability of $U_{0 \text { west }}$. Consequently, it is sufficient to investigate $U_{\text {0east }} \in \mathrm{R}_{\text {east }+}$ to know the linear stability of all the $U_{0}$.

\section{Acknowledgements}

Some of the data analyses and the visualizations in this paper were done with the ISPACK (http://www.gfd-dennou.org/library/ispack/), gt4f90io (http://www.gfddennou.org/libgary/gtool/), spmodel [16] (http://www.gfd-dennou.org/library/spmodel/) and the software products of the GFD Dennou Ruby project (http://ruby.gfddennou.org/). The numerical calculations were performed by the computer 
systems of the Institute for Information Management and Communication (IIMC) of Kyoto University and of the Research Institute for Mathematical Sciences, Kyoto University.

\section{References}

[1] M. Argentina, M. G. Clerc, R. Rojas, E. Tirapegui, Coarsening dynamics of the one-dimensional Cahn-Hilliard model, Phys. Rev. E. 71 (2005) 046210

[2] D. Bettinson, G. Rowlands, Stability of the one-dimensional kink solution to a general Cahn-Hilliard equation, Phys. Rev. E. 54 (1996) 6102-6108

[3] J. W. Cahn, J. E. Hilliard, Free energy of a nonuniform system. I. interfacial free energy, J.Chem.Phys. 28 (1958) 258-267

[4] A. Chekhlov, S. A. Orszag, S. Sukoriansky, B. Galperin, I. Staroselsky, The effect of small-scale forcing on large-scale structures in twodimensinal flows, Physica D 98 (1996) 321-334

[5] H-P. Huang, W. A. Robinson, Two-dimensional turbulence and persistent zonal jets in a global barotropic model, J. Atmos. Sci. 55, (1998) 611-632

[6] H-P. Huang, B. Galoerin, S. Sukoriansky Anisotropic spectra in twodimensional turbulence on the surface of a rotating sphere, Phys.Fluids 13, (2001) 225-240

[7] R. K. Kraichnan, Inertial ranges in two-dimensional turbulence, Phys. Fluids 10 (1967) 1417-1423

[8] L. D. Landau, E. M. Lifshitz, Quantum mechanics: non-relativistic theory, third ed., Oxford, Pergamon Press, 1977 pp. 73-74

[9] J. S. Langer, Theory of spinodal decomposition in alloys, Annals of Physics 65 (1971) 53-86

[10] A. J. Manfroi, W. R. Young, Slow evolution of zonal jets on the beta plane, J. Atmos. Sci. 56, (1999) 784-800 
[11] G. K. Vallis, Atmospheric and oceanic fluid dynamics, Cambridge, Cambridge University Press, 2006 p. 382

[12] A. Novick-Cohen, L. A. Segel, Nonlinear Aspects of the Cahn-Hilliard equation. Physica D 10, (1984) 277-298

[13] T. Nozawa, S. Yoden, Formation of zonal band structure in forced twodimensional turbulence on a rotating sphere, Phys. Fluids 9 (1997) 2081-2093

[14] K. Obuse, Takehiro S., and M. Yamada Long-time asymptotic states of forced two-dimensional barotropic incompressible flows on a rotating sphere, Phys. Fluids. 22 (2010) 156601

[15] P. B. Rhines, Waves and turbulence on a beta-plane, J. Fluid Mech. 69 (1975) 417-443

[16] S. Takehiro, Odaka, M., Ishioka, K., Ishiwatari, M., Hayashi Y.-Y., and SPMODEL Development Group, A series of hierarchical spectral models for geophysical fluid dynamics. Nagare Multimedia 2006, Available online at: http://www.nagare.or.jp/mm/2006/spmodel/

[17] G. K. Vallis, M.E.Maltrud, Generation of mean flows and jets on a beta plane and over topography, J. Phys. Oceanogr. 23 (1993) 1346-1362

[18] G. P. Williams, Planetary circulations:1. barotropic representation of Jovian and Terrestrial turbulence, J. Atmos. Sci. 35 (1978) 1399-1426

[19] S. Villain-Guillot, 1D Cahn-Hilliard equation: Ostward ripening and modulated phase systems, IJBC 10 (2009) 3541-3552 

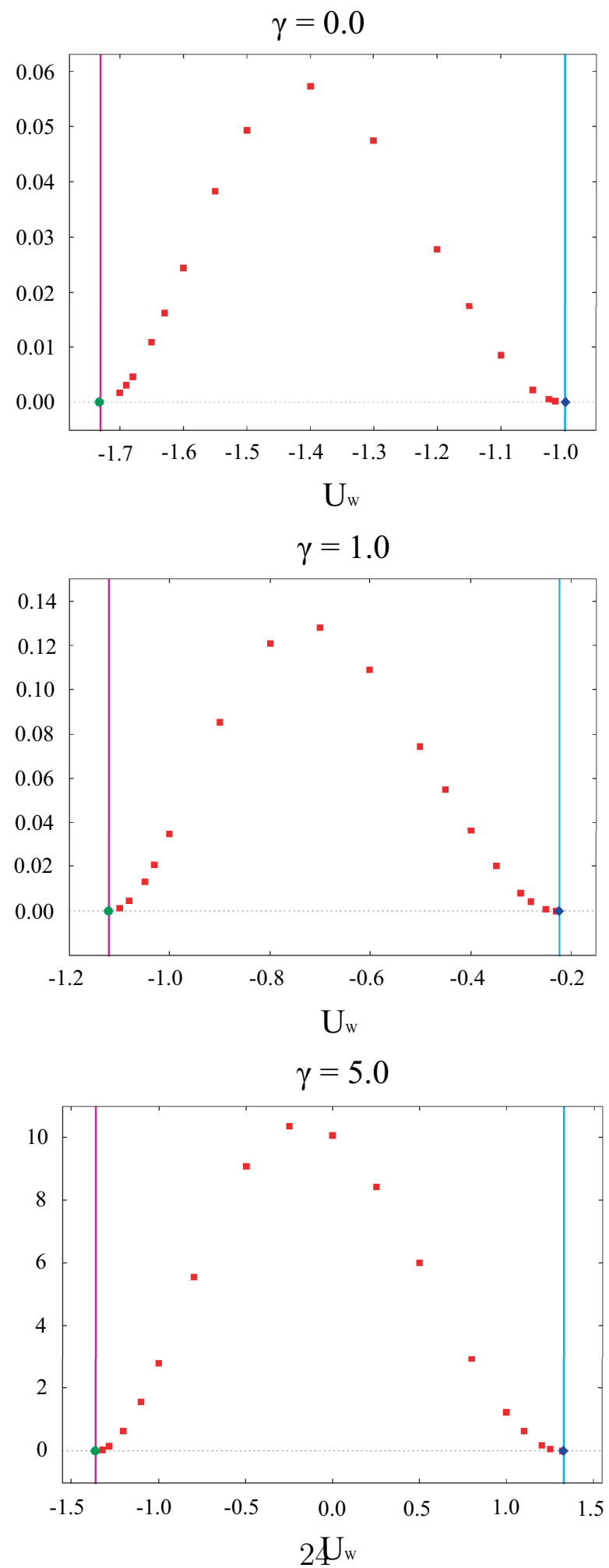

Figure 4: The real parts of the first eigenvalues (red squares) for $\gamma=0.0$ (top), 1.0 (middle), and 5.0 (bottom). The purple and the light blue lines denote the two boundaries (17a) and (19a), which also correspond to the purple and the light blue curves in Fig. 2. The green circle and the blue lozenge are the zeros obtained from the fitted lines shown in Fig. 5. 

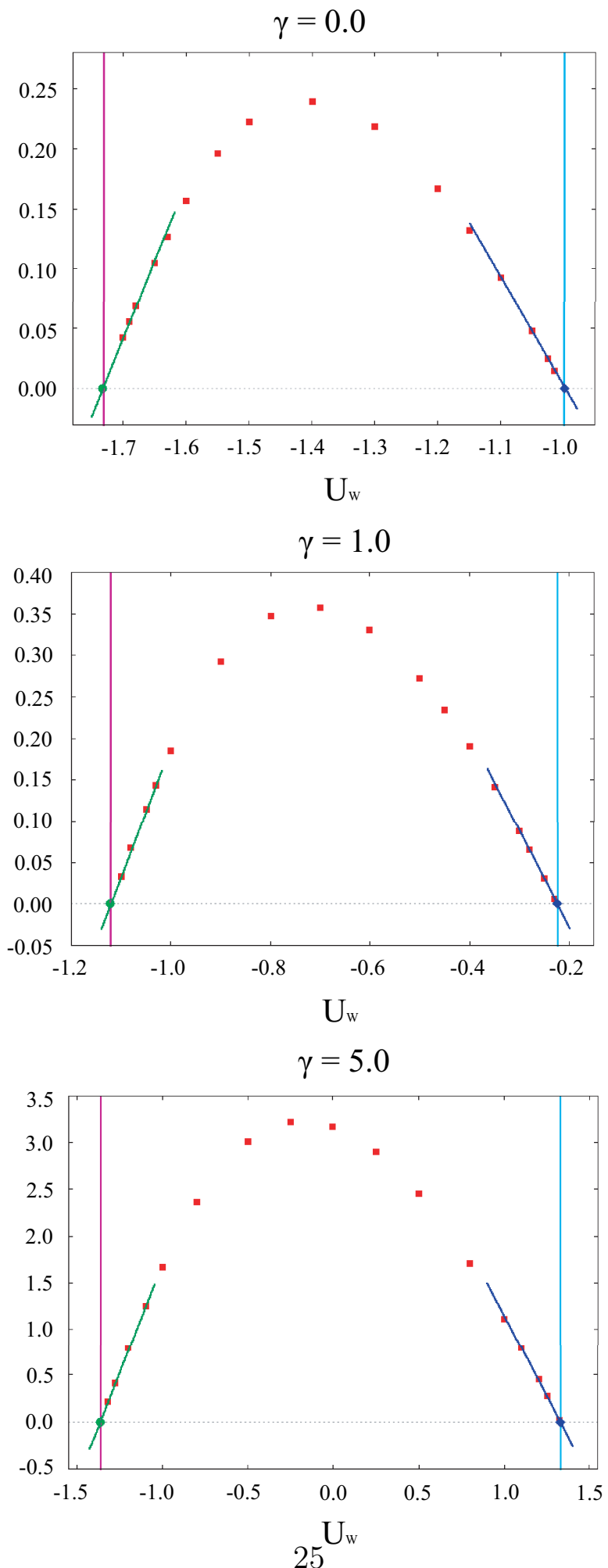

Figure 5: The square roots of the real part of the first eigenvalues (red squares) for $\gamma=0.0$ (top), 1.0 (middle), and 5.0 (bottom). The purple and the light blue lines denote the two boundaries (17a) and (19a), which also correspond to the purple and the light blue curves in Fig. 2. The dark green and the dark blue lines are the fitted lines from the data, and the green circle and the blue lozenge are the zeros obtained from these fitted lines. 

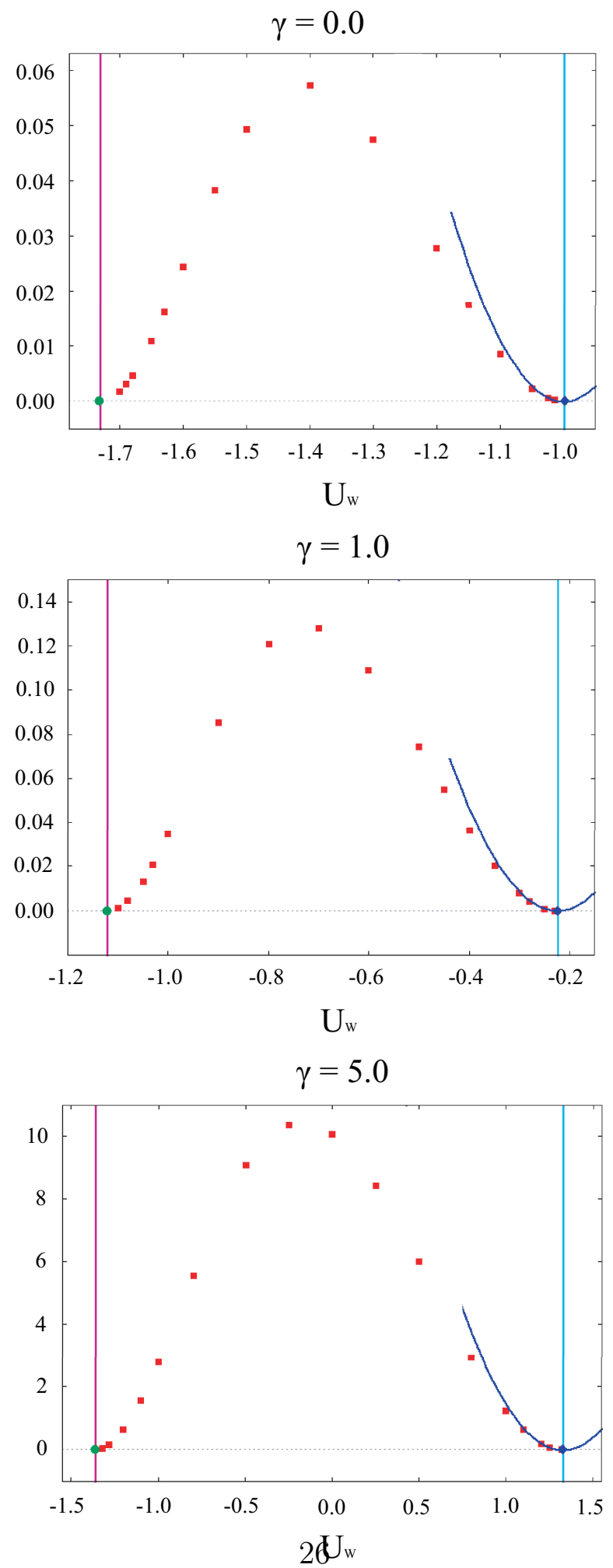

Figure 6: The real part of the first eigenvalues numerically calculated in $\S 4.1$ (red squares) and the analytical curve (34) (dark blue curves) for $\gamma=0.0$ (top), 1.0 (middle), and 5.0 (bottom). The purple and the light blue lines correspond to the two boundaries (17a) and (19a). The green circle and the blue lozenge are the zeros obtained from the fitted lines shown in $\S 4.1$. 

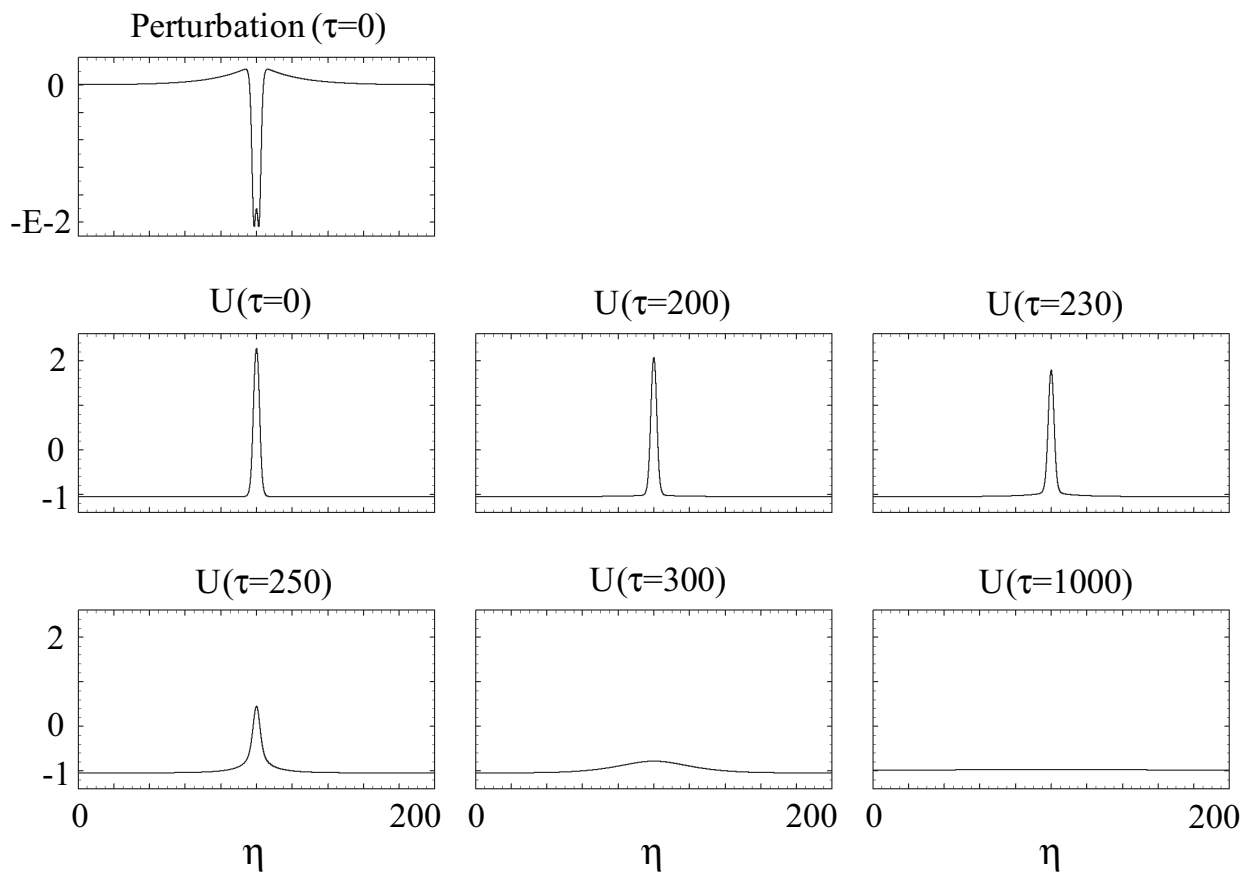

Figure 7: The eigenfunction of the first eigenfunction (top row) and the temporal variation of the $U_{0}$ added the eigenfunction as a perturbation (middle and bottom rows) for the case $\left(\gamma, U_{W}\right)=(1.0,-1.05)$. Each panel is at time $\tau=0,200,230$, (middle row, from the left to right) $\tau=250,300$, and 1000 (the bottom row, from the left to the right). 